\title{
Oration
}

\section{Secondary surgery of the flexor tendons}

\section{David Elliot}

St Andrew's Centre for Plastic Surgery, Broomfield Hospital, Chelmsford, England, UK

The R. Venkataswami Oration of the ISSH Nov 2004

W e have spent the last fifty years talking about primary flexor tendon surgery and very little thought has been directed towards secondary surgery during this time, although it is a large part of our flexor tendon workload. While primary surgery has moved on, secondary surgery has not and any discussion of this subject inevitably has to look back to the 1970s and 80 s for information on surgical technique, for scientific papers and for means of assessing the results, as very little has been written on this subject since then. The time has come to look critically at what we are doing under this banner and I would like to examine various aspects of the subject and see where improvements might be made.

Much of our flexor tendon surgery in Western Europe, as in the United States, is primary surgery. However, this is by no means universal and we do have to undertake a surprising amount of secondary flexor surgery, some of which is because of delayed presentation and some of which is because the flexor tendon injury quite frequently does not come as an isolated and simple one but in association with injuries to other tissues. The results of the initial surgery are also not universally good enough and secondary surgery for us is mostly that of the complications of primary repair, namely ruptured and stuck repairs. In the best institutes, this constitutes about $10 \%$ of all primary repairs. This is not a small number and something of an indictment of our present techniques of primary surgery. The cases which come to secondary surgery are mostly either the result of more severe injuries or have occurred in patients who make excessive amounts of scar tissue or have not co-operated with therapy because of low pain thresholds, social circumstances or stupidity. So the cases which I see needing secondary surgery are frequently either 'bad injuries' or 'bad patients'. The situation in some of your practices may not be so very different. However, many patients in India will only get to an appropriate surgeon at a time when secondary repair of the tendon using grafts is the only option because of proximal tendon retraction. This, by definition, becomes 'Secondary Flexor Surgery', although the problem is simply an extended finger with good passive but no active flexion, but now no longer amenable to direct repair. Unfortunately, among the patients presenting after delay - also defined as undergoing 'secondary flexor surgery' - are a group with much more complicated problems, sometimes, as a result of injuries to the other structures of the digits and, sometimes, as a result of the unaided healing process within a digit in the presence of an inactive flexor system. The problems in these cases are not simply those of being unable to get the tendon ends together. So, in terms of the pathologies in the digits themselves, the problems we all face in this rather heterogeneous mix of cases, which we call 'secondary flexor tendon surgery', are not so different, namely flexor tendons which are not intact and flexor tendons which are stuck in scar tissue, variably associated with divided pulleys, stiff fingers and injuries to other, adjacent structures in the fingers, hand or forearm.

Although the technique of tendon grafting may give comparable results to primary flexor tendon repair in the hands of an expert, the literature and general opinion would support the view that the latter is less difficult for most surgeons and more likely to be more successful, even if this has never been proved by comparative trial. For most of us, tendon grafting is more difficult, therapy is more vital to success and the results are usually less near to normal. A separate issue is the fact that the reported results of secondary flexor tendon surgery are generally worse than those of primary surgery. 
Undoubtedly, this relates, not only to surgical technique, but to the fact that we collect and report all secondary flexor surgery cases together. We are bundling together the complex cases which also incidentally, need a tendon graft with fingers which simply need a graft instead of a primary repair to reconnect the tendons.

While the mode of re-connection of the tendons may or may not be relevant, the other factors making secondary surgery less likely to achieve good results need to be analysed as some are amenable to changes to improve this situation. My secondary surgery group include my worst injuries and worst patients. They are likely to do badly whatever I do and may do no better after secondary surgery than they did the first time. For those of you doing less primary surgery, there will be, among your cases undergoing secondary surgery, the same proportion of bad injuries and bad patients. Psychological manipulation and time spent are the only weapons we have against these factors and we probably cannot change them more than a little.

It has generally been recognised over the last fifty years that limbs repaired immediately and moved early do better because they do not stiffen in scar tissue and do not develop contractures during healing as a result of inactivation of one or more of the normal parts of the locomotor system. This truism applies to the hand without flexor tendons. We can change this factor: primary, or early, surgery allows the patient to move the hand early, albeit with varying degrees of help, and negates some of the problems, at least some of the time in some of the patients. Surgery in scarred tissues is also more difficult and is less likely to be followed by good movement, even if mobilisation is immediate. These are problems we can avoid by doing more primary and less secondary surgery. Various techniques will allow us to make this switch.

The move towards more primary surgery, worldwide and whenever feasible, was largely a result of the pioneering work of Kleinert, Verdan and others in the 1950s. These surgeons introduced immediate repair and immediate mobilisation of acute injuries. In most parts of the world, the extent to which their methodology has been adopted remains a medico-political battle about the distribution of hand casualties and about training of more hand surgeons. Most of us, individually, only ever have limited influence on this. However, we can tilt the balance in our own practices, firstly, in favour of primary tendon repair and, secondly, to avoid our primary repairs failing and requiring further surgery. In our unit, we are now much more aggressive in our policy with respect to delayed primary repairs. If a patient comes late, and the finger is mobile passively, we explore the finger immediately and try to repair the tendons. If the tendon ends will not quite come together, we use the lengthening of the tendon within the muscle in the forearm, described in 1986 by Professor Le Viet of Paris, to achieve a primary repair. In this procedure, although the tendon has been cut within the muscle, the muscle itself has not and the muscle fibres maintain continuity of the musculotendinous unit, so allow immediate mobilisation. A single tendon cut gives about half a centimetre in extra tendon length distally. If one repeats this cut again, about 1-2 centimetres from the first cut but still within the muscle, the second cut will give another quarter centimetre of lengthening. If repair still proves impossible then a graft can be done with no loss of time or a silicone rod can be put in as the first stage of a two-stage tendon grafting procedure, according to preference. A paper written by McFarlane and his colleagues in Toronto in 1968 now has an importance in this respect, for reasons not appreciated at the time. Unintentionally, it showed that delayed primary repair is possible in more cases than we might believe even after very long delays. At that time, hand surgeons in North America were trying to get started with the Kleinert/Verdan philosophy of immediate repair and immediate mobilisation. However, hand units were still receiving patients at quite long times after the initial injury, presumably because the senders were expecting them to be treated by secondary grafting. McFarlane and his colleagues tried to do primary repairs in one hundred patients sent to them, whatever the delay. A number of these patients arrived more than twelve months after the initial injury. One was sent at seven years. In this case, McFarlane could get the tendon ends together but he felt the repair was a little tight so he undid the repair and used a tendon graft. Perhaps if Le Viet lengthening had been available then, he would not have abandoned the primary repair. That the divided flexors in 36 of 100 fingers could be repaired directly, even months after the injury, has great significance to us as it negates the assumption that delayed presentation necessitates tendon grafting routinely. Add Le Viet's tendon lengthening to these results and 36\% might have been higher. I think McFarlane's work needs repeating and it would be appropriate to do this further work in India, 
where delayed presentation is common.

When the FPL is cut, the muscle retracts more than the finger flexor muscles and primary repair is often difficult or even impossible after only two or three days. Either of the two French techniques of lengthening the proximal FPL tendon within the muscle can allow primary repair. In 1950, Rouhier simply cut the tendon out of the muscle and sutured it back again, but more distally in the muscle belly. I have already described the alternative Le Viet technique. The Le Viet's technique can also sometimes be useful in cut finger flexors or FDP pull-offs presenting late in which the finger flexor muscles have shortened and also in cases presenting with small segments of tendon missing.

When a profundus tendon is pulled-off distally or, more rarely, is cut in Zone 1 and drops back through the A4 pulley then presents late, it may be too swollen to pass through the pulley. Because the distal part of the profundus is double-barrelled, secondary surgery can be avoided using a technique we described in 2001. The distal part of the tendon is halved and one half discarded. The remaining half of the tendon is passed easily through the A4 pulley to reattach it distally. This is a reasonable thing to do as half a profundus tendon is thicker than a palmaris tendon graft harvested from the same hand. The half profundus tendon is also much easier to suture with conventional core sutures than a palmaris or plantaris graft. Halving of the tendon is normally only necessary as far proximally as the proximal interphalangeal joint but can be taken all the way back into the palm if necessary to allow a swollen profundus tendon to be past through the A2 pulley and superficialis decussation as well as through the A4 pulley.

We can take measures to ensure primary surgery leads less often to secondary surgery. It is sometimes forgotten that the Kleinert/Verdan plan had TWO parts. Immediate repair is likely to lead to secondary surgery unless followed by immediate mobilisation. In respect of the immediate mobilisation, I think the presentation to the Indian Society for Surgery of the Hand in 2002 by Dr. Sunil and his colleagues from Bangalore may have significance not only for you but also for the West. These researchers evolved a means of making Indian patients mobilise primary flexor tendon repairs in the manner first described in Belfast, without rubber bands, which I have used for twenty years and about which I have talked often in India. His results were as good as ours but with the patients doing the mobilisation themselves and with no therapists. After initial instruction, his patients simply move their hand, still in a dorsal plaster splint, through a series of simple activities of active flexion then passive flexion then active extension. They do this whenever they think to do it and are not doing something else and not according to a specific therapy regime of a fixed number of times a day as we do. Probably, the number of movements per day is similar. Many of you may have no or not enough therapists. In the West, we are running out of therapists because the Government Hospitals do not pay them enough. Consequently, they are all going into private practice where they do very little hand work. So we are all in the same boat and this method of postoperative mobilisation, or something along this line, may be the answer for all of us. If early active mobilisation is possible in India primary repair without secondary tenolysis of stuck tendons later is a reality, perhaps, even for some patients arriving late, given what I have just mentioned earlier about delayed primary repair.

Forty years ago, Kleinert and Verdan showed that it was possible, and preferable, to repair both flexor tendons at primary surgery and we continue to repair both flexor tendons under nearly every circumstance. In the 1970s, Boyes pointed out the problem of repairs sticking under the A2 pulley, which is the tightest part of the sheath. In 1994, Professor Tang from Shanghai re-discussed this problem and showed better results when only the profundus was repaired for those injuries under the $\mathrm{A} 2$ pulley in the part of the sheath which he called zone $2 \mathrm{C}$. In a study in 1999, our results did not agree with Tan's study in respect of simple flexor tendon divisions in Zone $2 \mathrm{C}$, which seemed to have results equal to those in the other parts of zone 2. However, single tendon repair, probably, has a role in more complex injuries. During the last ten years, I have had a number of patients with severe injuries of the distal palm or bases of the fingers in whom we repaired both tendons at primary surgery then had to perform a tenolysis later. In all of them, each of the two flexor tendons was so oedematous that it was double its normal size. These huge tendons were completely jammed under the A2 pulley. I had to remove the FDS tendons completely to get any movement through the A2 pulley. Tang's single tendon repair at primary surgery would have avoided this secondary surgery. So, we now only repair the profundus tendon in any injury in this area which is likely to produce significant oedema such 
as crush injuries, replants, revascularisations, distally based flaps of the palm or proximal phalanx, multi finger injuries, etc. The same problem arises in Zone 4 in the carpal tunnel, although severe injuries here are rarer. At this level in these larger injuries, we also only repair the profundus tendon. Although not common, one of the commonest Zone 4 injuries we see are machete attacks. When the patient puts his hands up to protect his face, the Ulnar border of the hand faces the machete. If it strikes proximally on the body of the hand, the blade may penetrate as far as the carpal tunnel to divide all of the flexor tendons.

Re-repairing ruptures of primary repairs also reduces the number of secondary procedures we have to do. Not all ruptures can be re-repaired but many can be if the patient returns within 48 to 72 hours of rupturing. Suitably warned at the time of surgery, most patients will realise what has happened and do return within this time period. The results of re-repair in the literature suggest that we should - whenever possible - try to re-repair ruptures and not leave them for secondary surgery. However, this literature is very small and mostly only reports a few cases on the end of a few papers on primary repairs. To try to get a better view of this, we have just finished looking at the results of about 40 re-repairs in zones 1 and 2 done in our unit over the last fifteen years. Only about $50 \%$ eventually had good or excellent results and five of the forty ruptured again. So, this general rule about immediate re-repair needs further analysis and may not be universal, particularly in the little finger where ruptures again were commonest and results of re-repair poorest in this series. This is true, at least, if the re-repairs are done as we did them initially, namely with the same suturing technique as we used for the primary repair. If the re-repair is done with one of the stronger suture systems about which so much has been written in the last ten years, these results may be better. Alternatively, perhaps we should be grafting some of these cases, particularly in the little finger.

Moving on to the actual surgery, the technical problems are limited in number, although these may present to us in various combinations and with very variable degrees of complexity of each component. The overall clinical picture sometimes seem horrendous, occasionally even insurmountable, when all the factors are seen in summation. The options of treatment when the tendons are not in continuity are delayed primary repair, one-stage flexor graft, two-stage flexor graft and no repair (buddy strapping to the adjacent finger). The last is mostly used, temporarily, while waiting for fingers to soften. It is also used, occasionally, as a long-term solution, usually in the elderly but, occasionally, in younger patients who tire of our failures. Whether we should be carrying out single stage or two stage tendon grafting, or a mixture of both, depending on the individual case, is a matter of opinion with little hard fact to support either side of the debate. I always use two stage grafting as I was brought up at a time, and in a place, where it was believed to give the best results. Sometimes, it seems that this is overkill, as when the sheath is not badly scarred. However, most of my cases for tendon grafting are failures of primary surgery who, as a group, are 'bad patients', as defined above. Although not the reason for doing two stage grafting when I started, I have come to realise that this technique may, particularly, suit these cases and my practice. The circumstances of Indian hand practice push you more towards single stage grafting. However, there may be circumstances where this expedient may be so unlikely to give a good result that two stage should be considered. Despite the preference for single stage grafting of Professor Venkataswami and your other senior surgeons, who, remember, have great skill in this, and the supportive feeling that the particular suppleness of the Indian hand, as compared to the Caucasian hand, allows single stage grafting more effectively, no one has worked out how to identify the 'bad patient', whether in India or England. It is also perhaps worth a thought that the two stage graft was introduced because of dissatisfaction with the results of the one stage procedure, albeit in America. When the scar tissue between the tendon and sheath is fine and diaphanous we talk of adhesions and two stage grafting looks to be overkill. Unfortunately, the scar is often more dense: we then talk, not of adhesions, but of scarring! Perhaps it might be better to stage the grafting in these cases.

With respect to the actual technique of joining tendons, I have little to add to the technique which we know as the Pulvertaft weave. It works well. One modification of the original technique which I do use, which is possibly not routine, is to use a continuous running suture, woven up the length of the weave then back to its starting point and knotted only once at this point, rather than using many separate sutures, which leave an array of knots and spiky suture ends along the surface of the weave. I believe that this must increase the friction of the weave and the likelihood of it catching. When using this continuous stitch, if the needle is going to cut a previous pass of the 
suture, it does this at the time and not later and one has to start again. However, this rarely actually occurs. At the other end of the graft, we have got rid of the button technique by passing the suture through the tuft of the distal phalanx instead, a very simple refinement which we described in 1996. The button is, mostly, not a problem, but can occasionally be troublesome. Pulvertaft obviously felt the same about the button as, in 1965; he described a technique of passing the end of the tendon graft through the proximal end of the distal phalanx and suturing it back to itself.

Tendons become stuck, or adherent, to their surrounds very easily and this aspect of secondary flexor surgery is perhaps one of the most underestimated parts of it. Simple statements, such as 'Tenolysis' and 'Tendon Graft', which we put on our operating lists are often a gross simplification of the surgery needed and reinforce our underestimation of the problem. Whether secondary surgery is being carried out after delayed primary presentation or for failed primary surgery, all of the tissues on the palmar side of the finger may be scarred to some degree and each layer may require treatment. Scarring of the skin and subcutaneous soft tissues may cause longitudinal skin shortening. A mid-lateral incision with a proximal palmar $\mathrm{V}$, which allows one to advance the $\mathrm{V}$ of skin from the palm into the finger is usually enough to deal with skin shortage where the original skin injury was a simple cut. We, usually, do not close the V as a Y, but allow it to epithelialise under a moist antiseptic dressing, done by the patient twice daily during the first few post-operative weeks of mobilisation, much as is comonly used for fingertip injuries without bone exposure or for the McCash open palm treatment of Dupuytren's disease. The palmar V wounds close over 2-4 weeks. Preoperatively, it is usually obvious, from the nature of the primary injury and/or the appearance of the finger, when more skin will have to be incorporated to achieve full extension. Where more skin is needed, a cross finger flap, taken at the level of the proximal interphalangeal joint from the finger adjacent to the injured finger on the side of the mid-lateral incision, can be let into a transverse incision in the palmar skin of the finger after it has been raised on the opposite side of the finger during exposure of the tendon sheath. This is an easy way of adding extra skin while using the same approach for moderate cases of skin deficiency. Worse cases need more elaborate flap reconstruction, often with distant flaps. While injuries with a skin deficit primarily are less common, it is almost universal for the subcutaneous tissues to show variable degrees of scar deposition in, or replacing, the subcutaneous fat. The scarring of the subcutaneous tissues prevents normal skin mobility and is the common reason why there is a skin shortage in a longitudinal direction.

As one raises the palmar skin of the finger from the midlateral incision, this is immediately obvious, as is the scar thickening of the tendon sheath. To get to the tendons, conventionally, we remove the entire sheath except the A2 and A4 pulleys. Without thought, we have released the sheath scarring in the longitudinal direction. For reasons which I will discuss later, I would recommend that the $\mathrm{A} 3$ pulley also be preserved during this exposure of the tendons.

Next, the actual tenolysis. This can be difficult to do without doing more damage than good, so needs time. Even the slightest small strand of fibrous tissue linking the tendons to one of the pulleys will stop movement. Once movement is stopped, secondary adhesions can form elsewhere along the tendon sheath. It is common at secondary surgery to see a primary repair firmly stuck under the A2 or A4 pulley, presumably the primary event, with less dense adhesions along variable amounts of the remainder of the tendon sheath. These are, presumably, a secondary event. It is particularly difficult to free the tendons under the pulleys on their dorsal surfaces and, although rarely admitted, division of a pulley at this stage - usually the A4 - is probably the commonest cause of pulley loss. When the tendons are very swollen and tight under the A2 pulley and seem likely to stick again, I remove the FDS without further thought.

The deepest problem, and the most likely to give rise to a recurrence of loss of extension are the ligaments of the underlying joints. The proximal interphalangeal joint may only require proximal release of the palmar (volar) plate. More often, the accessory collateral ligaments also have to be released and, if the scarring is more severe, releasing the true lateral ligaments, distally from the middle phalanx or proximally from the proximal phalanx, may be necessary to straighten the joint. I find anything short of complete excision of the palmar (volar) plate of a contracted distal interphalangeal joint does not work either to straighten the joint or to keep it straight in the long-term.

Even after releasing all of the structures within the finger and distal flexor system, the fingers may not straighten, 
because of shortening of the muscles in the forearm. If this is the case, I do a Le Viet lengthening of the tendons within the muscles in the forearm.

If a primary repair is likely to stick when not mobilised immediately, a secondary procedure, whether tenolysis or graft, will do the same. After tenolysis alone, there is no repair to protect and much more aggressive rehabilitation can be used. We start this the next day under continuous Bupivacaine block of the median and/ or ulnar nerves as an in-patient to achieve as much motion as possible as early as possible. There are a few papers from twenty years ago confirming that flexor grafts do better if mobilised early. The tendon unions of a secondary repair are very much stronger than those of a primary repair so early active mobilisation has less risk. We use exactly the same regime of immediate mobilisation after secondary surgery as we do after primary repair, which simplifies things for everyone in the unit. Although it is not strictly the remit of this lecture to discuss tendon transfers, we also immediately mobilise all of these from the day after surgery using appropriate splints worn between exercising activities, but mostly removed completely, with the patient sitting and the hand on a table, to do exercises several times a day. We never use special regimes, such as the Strickland Frayed Tendon Regime. If a tendon is so frayed after tenolysis that I do not think it will survive rehabilitation, I replace it with a tendon graft. The therapists are not then faced with a regime with which they are unfamiliar and with tendons which may snap under their care. This possibility may make rehabilitation too cautious in a group of patients in whom this is likely to be counterproductive and lead to further stuck tendons. All of these cases are treated with antibiotics for five days post-operatively as infection may lose the game by creating so much more oedema and fibrin glue that the tendons will adhere again.

Bowstringing is a relatively unusual problem, probably because a lacerating injury which is longitudinal or oblique enough to cut the A2 pulley while leaving at least one flexor tendon intact is not common. Occasionally, a severe crush injury with bursting lacerations of the skin, and usually a badly comminuted proximal phalanx, will include rupture of the A2 pulley. Occasionally, A2 division is a result of bad previous surgery. Except after surgical intervention, bowstringing virtually only occurs in the proximal finger and thumb. If you hold your index finger and flex the distal interphalangeal joint with the proximal interphalangeal joint held straight, you will realise why we never see absence of the A4 pulley alone giving rise to more distal bowstringing. The distal interphalangeal joint flexes too little and the length of free tendon is too short for distal bowstringing to occur. It requires that the whole length of the sheath distal to the A2 pulley to be missing to give rise to distal bowstringing. I believe that this happens most commonly because we start all secondary flexor surgery in the finger by opening the sheath through the A3 and C pulleys and, usually, removing them in the process. Then the A4 is either too weak after our tenolysis and snaps during early rehabilitation or tears during the dissection of the tenolysis. Distal bowstringing can be avoided by keeping part of the A3 pulley at the start of secondary flexor dissections. Although this pulley is very flimsy in the uninjured finger, it is usually considerably thickened in these cases. Proximal bowstringing in the presence of one or two intact flexors, or if single stage grafting is carried out, demands a pulley reconstruction which is strong enough to resist the tendon forces when mobilisation is started immediately. Probably the commonest reconstruction used is that advocated by Lister in which a strong tissue, such as the Palmaris tendon or Extensor Retinaculum, is passed round the bone several times and sutured to itself. Bearing in mind that many cases undergoing flexor surgery have poor results because the extensor tendons, bathed in fibrin in the oedema of the injury, tether to the underlying skeleton and overlying skin to a greater degree than do the flexor tendons in their sheaths, and are restricting even passive finger flexion, I have reservations about the Lister technique. Techniques which attach the new pulleys to the sides of the phalanx avoid invading the extensor space, so may be better in this respect. Possibly the new and smallest bone tags will prove of use to facilitate and strengthen these techniques of pulley reconstruction and persuade us to leave the extensor space inviolate. We much more commonly have a need for pulley reconstruction when we explore a flexor secondarily and come across a severe 'mess' of scarring. Sometimes, the last cut of the tenolysis, or the removal of a completely welded-in flexor, destroys what was left of a weakened pulley and,

Sometimes, it is simply impossible to undo the scar tissue. For us, this is obviously a situation which demands use of a two-stage tendon graft. The tendons being removed from the finger, or, if these are too poor, the one you do not intend to use later as the motor for the graft, taken from more proximally, can be used as material to reconstruct the pulleys. The tendon is split longitudinally 
and opened out, then turned through 90 degrees. This provides enough material to make as many pulleys as one wants. Because there will be no force on them for 3 to 6 months, the new pulleys can be simply sutured to the remnant edges of the sheath, to which they will be strongly bound by the time they have to hold a tendon back. This technique also avoids the use of extensor retinaculum to make pulleys, as suggested by Lister, which leaves an obvious scar on the very visible dorsum of the wrist.

The timing of secondary surgery is likely to have considerable effect on the result. A finger which is swollen, hard and red, as is often the case at presentation with moderate delay after the initial injury and almost always after a catastrophe following primary surgery, is not suitable for immediate secondary surgery. While it is tempting to yield to pressure from the patient to operate as soon as possible, the patient has to be told that it is not advisable to explore the finger immediately and that it has to lose its swelling and stiffness and become soft and supple again to maximise the chances of secondary surgery being successful. This can take three, six or more months, at least in Caucasian digits. If operation is done too early, it can be difficult to identify and separate the structures or to suture them and the hand often responds with a worse healing reaction and further adherence of all of the palmar soft tissues. The skin scars, the swelling in the finger and its hardness are good indicators of what is going on inside the finger.

I would like to consider how we assess these injuries, as we cannot communicate without an acceptable method of assessment. I believe we need to start again with respect to our assessment of secondary flexor surgery. In the past, the techniques of assessment used for secondary surgery, such as that used by Boyes in the 1970s, accepted a poor level of function as a reasonable result. This allowed for the fact that many factors at the time - the total injury, the surgery and the rehabilitation - militated against truly good or excellent results. Nevertheless, it also accepted a lower standard than has been shown to be possible by primary surgery. For the future, we need to assess our secondary surgery using comparable techniques to those we use to assess primary surgery, if we are to get any idea whether they are acceptable in modern practice. The TAM is the most appropriate of the primary assessment techniques for assessment secondary surgery if it involves flexor tendon grafting as the movement of all three joints need to considered. The same will be true for most tenolysis procedures. Some which are truly restricted to the finger may be more honestly assessed using either the first Strickland assessment, which excludes the metacarpophalangeal joint, or, very occasionally, our variation of this for zone 1 injuries, which also excludes the proximal interphalangeal joint from the assessment.

I would like to finish with a caveat. In 2004, there can be few cases in which amputation either in the finger or as a complete ray is justified after simple flexor tendon division. Apart from the cosmetic angle and the grip loss of even a single finger amputation, a single end neuroma of the cut digital nerves can render a hand, which was a nuisance, completely functionless and sometimes destroy the patient's livelihood. We only rarely need this operation. This lecture has been a résumé of conventional secondary flexor tendon surgery. Rather than contemplate amputation in your worst cases, I would commend that you consider the alternative of using a vascularised transfer of a flexor tendon with its gliding bed taken from the fore-arm on a distally based Ulnar artery pedicle, described recently in this book by Jean-Claude Guimberteau of France. 